\title{
La axiología de la cooperación como elemento sustentador del liderazgo cooperativo
}

\author{
José Eduardo de Miranda \\ Doctor en Derecho - Director General de Kheíron Educacional
}

Sumario: I. Presentación. II. Los valores cooperativos y el rescate de la identidad del Cooperativismo. III. La moral del Cooperativismo. IV. La eficacia de la cooperación. V. Comprendiendo el liderazgo. VI. La incidencia de la axiología de la cooperación en el ejercicio del liderazgo cooperativo. VII. ¿Quién es el líder cooperativo? VIII. La importancia del factor humano. IX. Los diez rasgos personales esenciales del líder cooperativo: 9.1. Autoconfianza. 9.2. Autocontrol. 9.3. Censo de equidad. 9.4. Planificación y conocimiento técnico. 9.5. Certeza en la toma de las decisiones. 9.6. Metas y superación de resultados. 9.7. Amabilidad. 9.8. Responsabilidad general. 9.9. Cooperatividad constante. 9.10. Coraje. X. Las once heridas del liderazgo: 10.1. Inhabilidad organizativa. 10.2. Mala voluntad para las gestiones sencillas. 10.3. Preocupación excesiva por el presupuesto de su trabajo. 10.4. Sobrevaluación de sus conocimientos. 10.5. Miedo de turbulencias y de la competencia. 10.6. Incapacidad creativa. 10.7. Egocentrismo. 10.8. Descontrol emocional. 10.9. Deslealtad. 10.10. Apego al poder. 10.11. Preocupación por sus títulos. XI. A título de conclusión: la gran responsabilidad del líder. XII. Bibliografía.

Resumen: Los valores cooperativos son el núcleo que ha permitido el rescate y la consolidación de la identidad cooperativa, necesaria a la formación de la verdadera moral cooperativa, cuya comprensión y respeto de las necesidades humanas representa la esencia del propio Cooperativismo. Por este sentido, y bajo un manto axiológico, el liderazgo cooperativo debe conyugar las condiciones relacionadas al conocimiento técnico del líder con sus atributos personales, derivativos de los rasgos de su personalidad, esenciales a la gestión de los procesos gerenciales y humanos. El líder cooperativo debe ser el modelo de hombre cooperativo, el hombre honesto, que respeta la personalidad del prójimo, las diferencias entre las gentes y la igualdad en la dignidad de todos.

Palabras clave: Valores. Cooperación. Liderazgo.

Abstract: Cooperative values are the core that has allowed the rescue and the consolidation of the cooperative, which is necessary to the formation of the true moral cooperative identity, whose understanding and respect of hu- 
man needs represents the essence of the cooperative movement itself. For this sense, and under an axiological mantle, cooperative leadership must combine conditions related to the technical knowledge of the leader with their derivatives, personal attributes of your personality traits, essential to the management of human and managerial processes. The cooperative leader must be the model of cooperative man, honest man, who respects the personality of our neighbor, the differences between people and equality in the dignity of all.

Key words: Values. Cooperation. Leadership. 
... puede decirse que la cooperación es más humana que el capitalismo, sin olvidar que si bien ello constituye aspecto esencial, de su misión de progreso social es, al mismo tiempo, una fuente de debilidad para la cooperación ya que su buen desempeño está subordinado, mucho más que en la empresa capitalista, a la calidad de los hombres que la nuclea. El sector cooperativo está, por lo tanto, obligado a mejorar por sí mismo esta calidad humana.

Georje Lasserre ${ }^{1}$

En homenaje al Doctor Cecílio José Prates y Marli Malta Prates, verdaderos líderes cooperativos en su medio

\section{Presentación}

El predominio de la especie humana ha representado una de las más grandes transformaciones operadas en el desarrollo de la naturaleza, y esta transformación se consuma cuando el Hombre descubre su importancia delante del medio, la importancia del medio en donde habita, y, por, supuesto la importancia de aquellos que comparten el medio con él². Es así que el Hombre abandona la individualidad y pasa a vivir de manera gregaria. Bajo el aspecto comunitario, el sistema económico de la colectividad prehistórica, por así decir, tiene como objetivo principal la producción de los medios indispensables para la existencia de las propias colectividades ${ }^{3}$.

Al verificar los buenos resultados de la unión de esfuerzos para el alcance de objetivos comunes, el Hombre inicia naturalmente las bases del esfuerzo cooperativo consciente y sistemático, que evoluciona coherentemente de acuerdo a la comprensión racional de sí mismo y de sus necesidades.

De esta manera, «la cooperación como forma de trabajo conjunto probablemente se dio desde la aparición del Hombre, ya a nivel de economía doméstica, ya en una dimensión más amplia, cuando se inició la división del trabajo. Es de suponer que la cooperación en sus inicios no se dio sólo por causas económicas, sino que el Hombre en razón de su instinto gregario buscó a sus semejantes para expresar

1 Lasserre, G., El hombre cooperativo. Buenos Aires: Intercoop Editora Cooperativa Limitada, 1980, p. 11.

2 Ostrower, F., Criatividade e processos de criação. Petrópolis: Vozes, 1978, p. 11.

3 Academia de Ciencias de la URSS, Manual de Economía Política, Ciencias Económicas y Sociales, 3. ${ }^{a}$ ed., México D.F.: Grimaldo, 1968, pp. 24-28. 
sus relaciones afectivas, para reproducirse, para defenderse de los peligros, y también para practicar los cultos religiosos, las competencias deportivas, etc. $»^{4}$.

En este sentido, el Hombre probablemente «le deba más al efecto del principio de la cooperación que a cualquier otro, en su evolución biológica y social. No sólo esto, sino que, sin la función del principio de la cooperación, de la sociabilidad y de la ayuda mutua, el progreso de la vida orgánica, el mejoramiento del organismo y el fortalecimiento de las especies resulta totalmente incomprensible» ${ }^{5}$.

La cooperación, por supuesto, es el sello de la proximidad del hombre que unifica con sus semejantes la «fuerza» necesaria al alcance de objetivos comunes ${ }^{6}$. A par de ello, y considerando el quehacer desarrollado por un conjunto de personas, bajo la forma jurídico-societaria-cooperativa, el presente artículo puntuará la noción general de liderazgo y los principales elementos del liderazgo cooperativo, estableciendo los rasgos esenciales del líder que actúa bajo los valores de la cooperación. Para ello se va presentar una idea general sobre la importancia de los valores cooperativos en el proceso de rescate y preservación de la identidad cooperativa. Asimismo, se va a explicar la moral cooperativa y la eficacia de la cooperación, además de las principales heridas en el ejercicio del liderazgo cooperativo.

\section{Los valores cooperativos y el rescate de la identidad del cooperativismo}

Vigilante a todos los cambios que el mundo está a sufrir en diferentes sectores, y consciente de la sensibilidad de una época enmarcada por el crecimiento de la conciencia de la independencia global de la humanidad, en la búsqueda de la solución de los problemas que asolan el Planeta, los cooperativistas reaccionaran en el sentido de clarificar y fortalecer las bases ideológicas que siempre han servido de norte del movimiento cooperativo?.

${ }^{4}$ Ramírez Baracaldo, B., Teoría y doctrina de la cooperación. Bogotá: Ciudec, 1989, p. 76 .

5 Borgadus, E.S., Principios y problemas del cooperativismo. México D.F.: Libreros Mexicanos Unidos, 1964, p. 21.

6 Aquí, la expresión fuerza es utilizada en múltiplo sentido y puede representar técnicas, conocimientos, estrategias...

7 Miranda, José Eduardo de Miranda, De la crisis de identidad al rescate de la génesis del cooperativismo. Madrid: 2012, p. 77. 
La reacción se concretó en el Congreso de Manchester, a través de la Declaración sobre la Identidad Cooperativa, elaborada en base a la nueva formulación de los principios cooperativos y en la enunciación de los valores que deben ser seguidos a lo largo de la acción cooperativa, por las entidades y por sus miembros.

A partir de la definición de los principios como las pautas mediante las cuales las cooperativas ponen en práctica sus valores, debemos señalar que su interpretación como patrón no sólo de acción, sino también de evaluación, les remite a los valores, que evidencian la más absoluta esencia del espíritu cooperativo ${ }^{8}$. Es decir, considerados como un todo, estos principios se vinculan con los valores en los que se basan y resumidos en la definición, indican lo que es singular respecto a las cooperativas, independientemente de donde se ubiquen.

Las cooperativas, a partir del Congreso Manchester, en el año de 1995, reencuentran su esencia y vuelven a ofrecer al hombre la oportunidad de desarrollar una reflexión inmaterial, solidaria, de preocupación con su compañero y con todos aquellos vinculados a su ambiente, incluso el extra-cooperativo. Refundida con su propia identidad, las cooperativas vuelven a su marco de acción inicial, y su verdadero objeto supera el económico, una vez que los principios y valores cooperativos le sirven concientizar la necesidad de una permanente búsqueda por la mejora de las condiciones socio-económico-morales del hombre.

\section{La moral del cooperativismo}

La principal tarea del Cooperativismo es sembrar el entendimiento entre los ciudadanos; ubicar su ejercicio empresarial, a través de la sociedad cooperativa, en el sentido más ventajoso al bien común; favorecer la solución de las dificultades, permitiendo nuevos caminos que conduzcan a los individuos al encuentro de la prosperidad; y participar del desarrollo educativo del ser humano, de forma que éste se vea involucrado en una existencia basada en la labor coparticipativa?

El Cooperativismo es un paradigma social que sirve de herramienta apropiada para el enfrentamiento de las necesidades y la insurrección contra la inmoralidad, la inhumanidad, la injusticia económica y social, contra el olvido de aquellos que detienen el poder económico. Este enfrentamiento deja patente que el Cooperativismo ha de fun-

8 Ibid., p. 77.

9 Ibid., p. 78. 
darse básicamente sobre valores determinantes de la autoayuda, la responsabilidad, la democracia, la igualdad, la equidad, la solidaridad, la honestidad, la transparencia, y la vocación social. Estos valores son puestos en práctica por unos principios que determinan que los fines de la Cooperación se extienden más allá de la promoción de los intereses económicos individuales de los cooperativistas, para contribuir con los valores sociales y morales de una sociedad que necesita el amparo del hombre cooperativo.

El Cooperativismo es condicionante de una actuación humana-empresarial, volcada a la dignidad y a la justicia, que determina un sistema de deberes y acciones que entidad e individuos han de llevar a cabo: uno en pro del otro, y ambos a favor de los demás, sin perjuicio de los intereses particulares del miembro y de la empresa.

Hoy por hoy el Cooperativismo se encuentra a merced de una tarea trascendental que implica la acción de comprender y respetar las necesidades humanas; de atender a las necesidades de iniciar y ayudar a los individuos a seguir el camino del progreso personal, de la promoción, de la expansión y del perfeccionamiento del factor humano de cada persona. ¡Esta es la moral cooperativa! ${ }^{10}$

\section{La eficacia de la cooperación}

Ha sido en cooperativa vida tribal o social avanzada como nace el lenguaje, y luego la escritura, entonces sorprendentes medios de comunicar y de comulgar en propósitos comunes ${ }^{11}$.

Aranzadi manifiesta que la cooperación «no sólo es una práctica antiquísima, sino una conducta que se observa en todas las épocas, hasta las más remotas de la historia humana. Se conocen en todas las latitudes y tiempos diversas formas primitivas de cooperación de carácter familiar, tribal, etc. $»^{12}$.

Además, "estas costumbres e instituciones y su persistencia contienen una enseñanza más general, ya que patentizan el instinto y la tradición de ayuda mutua, de acción común, de posesión y de gestión en

10 Miranda, 2012, p. 84

11 Argueira Miranda, M.A., Carácter revolucionario del cooperativismo. Buenos Aires: Intercoop Editora Cooperativa Limitada, 1965, p. 17.

12 Aranzadi, D., El arte de ser empresario hoy: hombres y mujeres ante el reto de dirigir empresas cooperativas en el umbral del siglo xxı. Bilbao: Departamento de Trabajo y Seguridad Social del Gobierno Vasco, Federación de las Cooperativas de Trabajo Asociado de Euskadi, Universidad de Deusto, 1992, p. 258. 
común que se manifiestan en la conciencia y en la vida popular de todas las épocas y países. En efecto, no es nada extraño que haya sucedido, ya que el hombre es un ser intrínsecamente social. Aparte de las tendencias naturales a la sociabilidad, el hombre es un ser indigente, que no se basta a sí mismo, y se siente impelido a asociarse con los demás hombres para la defensa de sus derechos y a la satisfacción de sus necesidades» ${ }^{13}$.

Desde el momento que sufre la mutación morfológica que le imprime el aspecto efectivamente humano, la acción cooperativa se va gradualmente desarrollando en los diferentes niveles de la propia vida humana. En el principio surge como acciones reflejas, y posteriormente, de acuerdo a la complejidad, asume las características del instinto humano.

Es verdad que en el comienzo del proceso de evolución de la especie humana, la cooperación corresponde la consecución de unos objetivos relacionados con la supervivencia de un grupo; pero también lo es que se va manifestar en los periodos posteriores de la evolución humana bajo tipos comportamentales significativos de superación, para los cuales la existencia de unos cuantos individuos queda organizada en un cuerpo de personas que se unen con el objetivo de ayudarse mutuamente, uno con los otros, y todos con los demás.

Por tanto, la cooperación verdadera está en el comportamiento de aquél que pasa a interesarse colaborativamente por la vida de los demás, e intenta auxiliar en el proceso de transformación del Hombre, como un todo: social, económico, afectivo, y moral.

Esto ha sido lo que la historia ha mostrado, ya desde el inmediato momento en que el homínido se expone al proceso de desarrollo de la especie humana. Los individuos dejan de actuar en solitario y empiezan una labor conjunta para la satisfacción de unos intereses que en el principio son de uno, pero que visto con amplitud, son de todos.

La cooperación una forma de hacer, una actividad y un efecto, un talante de comportamiento social, y hasta mismo un modo de vivir que presume una relación corporativa y que tiene como objetivo un fin último, arraigado en un interés práctico de alcance de un bien común. Quizá por esto, estudios más profundos han examinado la Cooperación como norma ética, como proceso social y como estructura institucional.

13 Aranzadi, 1992, p. 258. 


\section{Comprendiendo el liderazgo}

Ya hace algunos años que el estudio del liderazgo está poniéndose de actualidad, «no como una moda más en la dirección de las empresas, sino como algo que va a tener duración y permanencia» ${ }^{14}$. Pero ya no como estudio de investigación puramente académica, que tuvo su época floreciente, sino «como convicción de su necesidad y aprendizaje del ejemplo de los líderes que hoy día aparecen en empresas sobresalientes» ${ }^{15}$.

El liderazgo, en líneas generales, es una condición humana, por la cual los atributos técnicos, de gestión, son complementados por rasgos de personalidad que facilitan el envolvimiento personal, necesarios para «educar, orientar y estimular las personas a persistieren en la búsqueda de mejores resultados en un ambiente de riesgos e incertidumbres» ${ }^{16}$. La idea de liderazgo transciende, por tanto, la noción singular y exclusiva de conocimiento técnico para el gerenciamiento burocrático, pues se conforma con la integración de elementos de aspectos personales inherentes a la personalidad del individuo.

Por así decir, el liderazgo comprende un proceso por lo cual una persona dirige, con responsabilidad, las actividades laborales individuales o colectivas (de uno o varios grupos) e interviene en ellas para ofrecer su apoyo, sagacidad, capacidad, reconocimiento de los aciertos y comprensión de los fallos generados por los individuos que están a su lado, con él.

\section{La incidencia de la axiología de la cooperación en el ejercicio del liderazgo cooperativo}

Es importante subrayar que los valores enumerados a lo largo de la Declaración sobre la Identidad Cooperativa reflejan los ideales trazados por los precursores, a través de las iniciativas que han apuntado a una cooperación que, además de solucionar las necesidades materiales del hombre, se ha preocupado de colaborar con su transformación moral.

Por esto es significativo acreditar que el ánimo de los precursores del Cooperativismo es fundamental, sigue valiendo y nada ni nadie

14 Aranzadi, Dionisio, El arte de ser líder empresarial hoy. Vitoria: Federación de Cooperativas de Trabajo Asociado de Euskadi, 1995, p. 19.

15 Ibid., p. 19.

16 Macedo, Ivanildo Izaias de e outros, Aspectos comportamentais da gestão de pessoas, 9 ed. Rio de Janeiro: Editora da FGV, 2007, p. 109. 
debe hacer que se les olvide, separándolos de la acción cooperativa por ningún motivo. De esta manera, se tiene en evidencia que los valores están presentes en el comportamiento humano, pues todo ser humano en su manera de pensar, sentir y actuar está bajo la influencia de determinados valores, que se manifiestan cuando la persona determina sus preferencias o define lo que le parece más importante ${ }^{17}$.

Estructurados bajo una jerarquía de preferencias, los valores son objetivos y a su vez tienen subjetividad, o sea, una misma opinión puede ser interesante para unos y despreciada por otros.

En el ámbito del Cooperativismo, los valores han perdido esta propiedad objetiva-subjetiva, puesto que la intención ha sido la de objetivizarlos, haciéndose con que todos tengan importancia idéntica entre cada persona que forma parte del movimiento cooperativo. Es decir, los valores cooperativos representan la identidad en la forma como las cooperativas y los cooperativistas deben abordar los problemas con los que se enfrentan continuamente, en el ámbito interno de la estructura económicamente organizada, y en el externo, en la órbita de los intereses de la propia humanidad. Los valores integran el espíritu cooperativo, como motor que impulsa el Cooperativismo vinculado a normas éticas y que tiende a alcanzar valores espirituales ${ }^{18}$.

Sintetizando esta idea, Aranzadi explica que en cuanto valor, el espíritu cooperativo incluye conceptos relacionados con el esfuerzo propio y la ayuda mutua serían actitudes de los socios de responsabilidad propia y de colaboración; con la idea de solidaridad y la igualdad entre los socios les lleva a procurar no sólo defenderse a sí mismos y proteger sus propios intereses, sino también defender la persona y proteger los intereses de sus coasociados; y a sentir que los problemas de los demás merecen ser atendidos de la misma manera que los suyos; la noción de justicia en las normas, la libertad y la equidad son disposiciones de los asociados para aplicar normas justas, entre sí y con terceros, sin privilegios arbitrarios, con la libre manifestación y ejercicio de su voluntad y de la misma cooperativa; la promoción humana y educación cooperativa con un profundo respeto a la dignidad personal y un interés por la mejora de la calidad humana de los asociados; y, por fin, y sin agotar todos los aspectos implícitos, la coincidencia con los intereses generales de la comunidad ${ }^{19}$.

17 Miranda, 2012, p. 62.

18 Ibid., p. 63.

19 Aranzadi, D., «Los valores cooperativos en el umbral del año 2000», en Anuario de Estudios Cooperativos 1993. Bilbao: Universidad de Deusto, 1994, p. 117. 
Bajo esta perspectiva, el ejercicio del liderazgo cooperativo debe suplantar los predicados únicamente relacionados a los tramos burocrático-administrativos de la sociedad cooperativa y debe, también, pautar una conducta de participación del líder, en el sentido de desarrollar una influencia completa sobre todas las personas que integran la entidad, influenciándolas positivamente, de manera que hagan suyos los valores del Cooperativismo, tornándose participes activos tanto de la búsqueda por los objetivos de la propia sociedad cooperativa, como del proceso de transformación permanente del hombre.

Los valores cooperativos, por tanto, deben integrar el perfil de los líderes cooperativos, conformando su capacidad ejecutiva con su habilidad personal para el trato con proyectos y el relacionamiento con personas.

\section{VII. ¿Quién es el líder cooperativo?}

Vigilante a la génesis de la identidad cooperativa, el líder cooperativo hoy es la persona hibrida, constituida de los conocimientos técnicos necesarios a la buena administración de la entidad cooperativo y contaminado por la esencia del espíritu de la cooperación, para que pueda inyectar en sus liderados el entusiasmo cooperativo y el deseo de colaboración con la entidad, con sus miembros y con el entorno.

El líder cooperativo es aquel que tiene conciencia de que el fin del Cooperativismo es el hombre en sí mismo, y el ejercicio de la sociedad cooperativa comprende el medio adecuado para el alcance de una transformación completa del hombre, en su aspecto social, económico y sobre todo moral.

En el ambiente de la entidad cooperativa, el líder es la persona que presenta capacidad absoluta para actuar positivamente ante los constantes cambios socioeconómicos que, muy rápidamente, afectan distintas estructuras organizadas fragilizando entornos, inclusive las cooperativas y el medio donde se encuentra inserida. El líder es el que actúa sobre la columna de los valores del cooperativismo, mostrándose apto para la solución de conflictos y pronto para el desarrollo de estrategias gerenciales, de administración de procesos humanos y administrativos.

El líder no puede, bajo cualquier aspecto, perder el hilo ideológico que sostiene el factor humano de la cooperación como el inicio y el fin del propio Cooperativismo20. "El líder debe tener perspicacia para dirimir en cada momento la tensión entre la libertad y las limitaciones: Cuándo

20 Macedo, 2007, p. 110. 
delegar y cuándo decidir uno mismo; cuándo «potenciar» a los seguidores y cuando usar del poder; cuándo escuchar y cuándo hablar; etc. La cuestión es fijar el grado de libertad y el grado de limitaciones, que debe tener en dado momento, sea la institución, sea la tarea del día» ${ }^{21}$.

El líder es aquel que está siempre animado por el espíritu cooperativo.

\section{La importancia del factor humano}

No es exagero decir que el líder es el responsable directo por el suceso de la sociedad cooperativa y por la felicidad de sus miembros. El líder, más allá de la absoluta preocupación gerencial, debe conectar su administración al factor humano interno y externo de la sociedad cooperativa, sirviendo indistintamente tanto a los asociados como a las personas que totalizan el entorno donde se encuentra inserida la entidad.

Siguiendo la orientación inherente a los valores del Cooperativismo, el líder tiene que ser paradigma de solidaridad, colaborando con el desarrollo total de todas las potencialidades de sus pares, a empezar por su desapego de la materialidad, para que pueda caminar de encuentro a su autorrealización como persona, persiguiendo constantemente el alcance de los atributos de aspecto ético-moral, afectivo, sensorial, intelectual, estético y también económico.

\section{Los diez rasgos personales esenciales del líder cooperativo}

Visto la importancia de los valores cooperativos en el proceso de rescate y preservación de la identidad cooperativa, el líder debe contornar su liderazgo bajo el manto axiológico del Cooperativismo, e, independiente de otros, debe tener en cuenta los siguientes atributos:

\subsection{Autoconfianza}

La autoconfianza deriva del conocimiento que el líder tiene de sí mismo y del encargo que ha asumido, de forma que acredita indudablemente en su capacidad técnica y en su condición humana. Bajo este aspecto, es importante decir que nadie, en ningún ambiente, se atreve

21 Aranzadi, 1995, p. 298. 
a ser liderado por alguien que no crea en si propio, o que pase a los demás incertidumbre respecto a sus potencialidades para la buena administración de procesos y gestión de conflictos.

\subsection{Autocontrol}

El autocontrol es el dominio absoluto de las emociones personales, que permite el hombre controlar a sí mismo, de manera que todas sus decisiones se establezcan razonadamente y no subjetivamente. El líder, de este modo, debe razonar siempre antes de la toma de decisiones.

\subsection{Censo de equidad}

El censo de equidad es la capacidad que el líder tiene de ajustar su planificación y normas institucionales a las distintas situaciones que valoricen el aspecto humano de sus liderados, bajo una condición legitima de justicia e de igualdad.

\subsection{Conocimiento técnico y planificación}

Mientras el conocimiento técnico significa la comprensión sistémica de las distintas situaciones y elementos que totalizan el ambiente de su actuación, la planificación establece la organización de los quehaceres, fortaleciendo el desarrollo del trabajo y ampliando los indicadores de seguridad en el ejercicio de la actividad de sus liderados.

\subsection{Certeza en la toma de las decisiones}

La buena comprensión de la realidad de su entorno y la planificación de su labor permiten el líder analizar las alternativas y mensurar sus resultados, estableciendo un sistema de gestión en que la toma de decisiones se cumpla con el máximo de certeza posible.

\subsection{Metas y superación de resultados}

Para establecer una relación de confianza y creencia con sus liderados, el líder debe saber cuál es el blanco de su actuación, definiendo 
muy visiblemente los resultados que desea superar. Las metas, por tanto, deben ser posibles de superación y su búsqueda debe ser orientada y fiscalizada, para que los orientados sientan seguridad en el proceso y en la ejecución de sus propios trabajos. Las metas no pueden ser utópicas. Los objetivos perseguidos por el líder deben ser posibles y pautados en un plan de trabajo apropiado para que los liderados no malgasten energía y labor, trabajando desorganizadamente y sin resultado positivo.

\subsection{Amabilidad}

La amabilidad es la cordialidad que el líder destina a sus liderados, mostrándose una persona comprensible, simpática y dispuesta ayudarlos en la solución de los problemas que surgen en el cuotidiano de trabajo y no abandonándoles en las situaciones particulares en las cuales necesitan, en muchas veces, un palabra de experiencia y de sabiduría.

\subsection{Responsabilidad general}

El verdadero liderazgo es reflejo de una responsabilidad general, por la cual el líder asume los riesgos y responde por ellos, sean directamente relacionados al ejercicio de su función o sean resultantes de actividad desarrollada por sus liderados. El líder jamás malgasta su tiempo buscando culpables por eventual problema y él mismo asume la responsabilidad por todas las complicaciones que puedan surgir en su cuotidiano. Todo el fallo cometido por cualquier de los liderados impone la necesidad del líder asumir la responsabilidad por el resultado, considerando suyos los fallos que han obstruido el desarrollo del proceso.

\subsection{Cooperatividad constante}

El líder debe ser un colaborador nato y debe estar pronto para cooperar con todos que están a su vuelta. La cooperación, por cuanto valor, debe ser utilizada por el líder como modelo de actuación y ejemplo constante para que todos sepan que co-operar, u obrar en conjunto, es la vía más adecuada para la unificación de esfuerzos con el propósito de superación de metas que siempre son de todos. 


\subsection{Coraje}

El líder cooperativo debe delinear su perfil dentro de un contorno de arrojo, fundamental para el desarrollo y preservación del manto ideológico del Cooperativismo y preponderante para la toma de todo el tipo de decisiones.

\section{Las once heridas del liderazgo}

Para preservar los diez rasgos principales al ejercicio del liderazgo cooperativo, el líder no puede ser acometido de las siguientes heridas.

\subsection{Inhabilidad organizativa}

El liderazgo cooperativo eficiente exige plena capacidad organizativa del líder, permitiéndole conocer el camino a ser trillado y preparándole paras las sorpresas que podrán surgir en la caminada. Ningún líder, bajo cualquier aspecto, puede negarse a lo que sea, justificándose ocupado demás para hacerlo. La negativa al cambio de la ruta por cuestiones personales es perjudicial al liderazgo y a la imagen del líder. Por esto el líder debe tener buena capacidad de organización, para que conozca el trazado a ser cumplido y pueda, siempre, ajustarse a los imprevistos.

\subsection{Mala voluntad para las gestiones sencillas}

En el ejercicio del liderazgo el líder no puede privilegiarse con las mejores tareas, delegando a los demás las más difíciles o eximiéndose de hacer aquellas más sencillas o de menor proyección en el ambiente de trabajo. El buen líder esta pronto para todo, incluso para ejecutar también actividades que delegaría a otros.

\subsection{Preocupación excesiva por el presupuesto de su trabajo}

El liderazgo presupone dedicación, identidad y envolvimiento. Por esto, el líder no trabaja por dinero, sino para el alcance de una meta o búsqueda de un ideal. De esta manera, el liderazgo no puede, jamás, conyugarse con el precio del sueldo o al valor de las comisiones que puedan ser recibidas por las metas alcanzadas. 


\subsection{Sobrevaluación de sus conocimientos}

La humildad es una de las claves del liderazgo de excelencia. El líder debe reconocer fortalezas y fragilidades de su equipo, y nunca, jamás, pude sobrevaluar sus conocimientos, poniéndose en condición de dueño de la verdad y señor de la sabiduría.

\subsection{Miedo de turbulencias y de la competencia}

El miedo es una de las peores heridas que puede afectar el liderazgo. Por tanto, el líder, para ser efectivo y respetado, no puede temer nada ni a nadie. El líder jamás podrá temblar ante obstáculos desconocidos o flaquear a las sospechas de que alguien desea ocupar a su sitio. Por esto, el líder debe estar preparado tanto para superar todos los tipos de adversidades como para mantener con sus liderados una relación de respecto y acreditación, entrenándoles inclusive para sustituirle en razón de causas presumibles o hechos desconocidos.

\subsection{Incapacidad creativa}

El liderazgo sujetase a imprevisiones y necesidad de ajustes de los procesos técnicos o de la gestión relacionada con las cuestiones humanas. De ese modo, el líder debe poseer buena imaginación, para que pueda conducir las adaptaciones necesarias a la búsqueda de las metas o al alcance de los objetivos. Un líder sin imaginación, o sin capacidad creativa, es incapaz de enfrentar las urgencias del cuotidiano y tiene su credibilidad comprometida, pus su poca creatividad puede oprimir los procesos.

\subsection{Egocentrismo}

Jamás el liderazgo pude ser marcado por la huella del egocentrismo, de manera que el líder nunca debe asumir la responsabilidad y el honor por las conquistas de sus liderados. La realización del líder es el éxito en las metas, el crecimiento de las personas, la transformación positiva del entorno y no los premios por los resultados. Por tanto, el líder dispensa los honores personales, reconoce la labor del equipo y recompensa todos los éxitos, sin desacreditar los que fallan. 


\subsection{Descontrol emocional}

El líder, para mantener la armonía de su labor, debe dominar muy bien sus emociones, controlar sus impulsos y conocerse a sí mismo, de manera que no se muestre una persona desesperada, nerviosa e incapaz para superar situaciones negativas.

\subsection{Deslealtad}

El liderazgo que no se establece sobre una columna de lealtad común, entre las personas que integran un equipo o un grupo, será víctima de turbulencias irrecuperables que afectarán la credibilidad y la posición del líder. La deslealtad es una de las principales causas por las cuales las personas no alcanzan el suceso.

\subsection{Apego al poder}

El liderazgo no resuelta de poder o de autoridad, sino que es derivado de la intervención de una persona dotada de calidades diferenciadas para la conducción de procesos y para el trato con las gentes. Por ello, el líder no puede ocultarse tras el sello del puesto y utilizar de la fuerza para hacer con que su equipo haga lo que determine como adecuado para la búsqueda y alcance de los objetivos. El uso del poder es indicio de inseguridad, pues un buen líder debe marcar su presencia con actitudes humanas, de simpatía, comprensión, lealtad, justicia y solidaridad.

\subsection{Preocupación por sus títulos}

De la misma forma como el poder, el título puede ser, también, una trampa para el éxito del liderazgo. El líder competente no se oculta tras sus títulos, pues el trato que tiene con la gente es de igualdad, priorizando siempre el respeto y la consideración recíproca.

\section{A título de conclusión: la gran responsabilidad del líder cooperativo}

Ya resulta evidente que «el poder de decisión que la cooperación le atribuye a la persona y no al dinero, le permite expresar su satisfac- 
ción (que es demasiado compleja para que se la pueda cuantificar directamente) o sus críticas y orientar consecuentemente la acción de su empresa» ${ }^{22}$.

En él ámbito de la entidad cooperativa, la interposición humana es completa, pues participa de la determinación de su objetivo e interviene como medio para el éxito económico.

Como consecuencia de la extensa participación de lo humano en el seno de la cooperativa, sus miembros establecen relaciones personales que hacen de ella una asociación entre partes. "Y la vida de esa asociación, más o menos intensa, más o menos armónica, orientada en tal o cual sentido, ejerce influencia sobre el funcionamiento de la empresa y su eficiencia» ${ }^{23}$. Es decir, el talante intensamente humano de la cooperación consiste en una grandeza que complica las cosas, pero cuyo valor es incalculable.

Por esto, para el buen ejercicio del liderazgo cooperativo, el líder debe tener en cuenta que su actuación se encuentra astricta a la propia naturaleza de la sociedad cooperativa, una típica «asociación de personas que se han unido de forma voluntaria para satisfacer sus necesidades y aspiraciones económicas, sociales y culturales en común, mediante una empresa de propiedad conjunta y gestión democrática» 24 .

Consciente de que las cooperativas son entidades jurídicas que tienen el fin primero de servir a sus asociados, el líder cooperativo debe difundir la importancia de la cooperación desarrollada bajo su matiz axiológico, y, así, establecer una sólida conexión entre los intereses de los socios cooperativos y de la propia sociedad con las diferentes necesidades de la comunidad externa, unificando, de este modo, los impulsos humanos necesarios a la satisfacción de los intereses económicos-sociales y al alcance de la transformación moral del individuo en sociedad.

Lograr una satisfacción socio-económica y conseguir la transformación moral del individuo solamente será posible en el momento que el líder cooperativo se percate de la necesidad de propagación de los valores cooperativos en el seno comunitario, lo que conseguirá mediante el fomento de los principios de la educación, formación e información,

22 Lasserre, 1980, p. 13.

23 Ibid., p. 12.

24 ACl, Declaración de la Alianza Cooperativa Internacional sobre la Identidad Cooperativa aprobada en Manchester. Consejo Superior de Cooperativas de Euskadi, Vitoria-Gasteiz, 1996, p. 17. 
e interés por la comunidad 25 . Es de esta manera que se va a consolidar la moral cooperativa, bajo la divulgación y aplicación de los valores esenciales, como la dignidad de toda persona humana y el respeto que se le debe.

El líder cooperativo tiene la responsabilidad por el estabelecimiento de todos los mecanismos necesarios a la mejora de la relación entre los individuos que integran la entidad cooperativa, acentuando así un valor de decencia global que es legítimo en todos los tiempos, en diferentes situaciones, y para todas las regiones del Globo. Es por esto que al velar por los intereses de los miembros de la entidad, el líder debe ser consciente, siempre, que le corresponde el deber de promover la educación en el seno de la comunidad en donde se asienta la cooperativa, y busca alcanzar una transformación completa del hombre que representa la base del nacimiento, desarrollo y existencia del propio Cooperativismo ${ }^{26}$.

Tiene el líder la responsabilidad de «mantener el rumbo e inspirar a otros que trabajen en colaboración mutua hacia una visión compartida de futuro. El líder creíble no sólo siente estas tensiones, sino que debe aprender a amar la lucha, en el afán de conducir las energías en la dirección deseada» ${ }^{27}$.

\section{Bibliografía}

Academia de Ciencias de la URSS: Manual de Economía Política, Ciencias Económicas y Sociales. 3. ${ }^{a}$ ed., México D.F.: Grimaldo, 1968.

ARANZADI, D.: El arte de ser empresario hoy: hombres y mujeres ante el reto de dirigir empresas cooperativas en el umbral del siglo XXI. Bilbao: Departamento de Trabajo y Seguridad Social del Gobierno Vasco, Federación de las Cooperativas de Trabajo Asociado de Euskadi, Universidad de Deusto, 1992. AranzADI, D.: «Los valores cooperativos en el umbral del año 2000», en Anuario de Estudios Cooperativos 1993. Bilbao: Universidad de Deusto, 1994.

AranzADI, Dionisio: El arte de ser líder empresarial hoy. Vitoria: Federación de Cooperativas de Trabajo Asociado de Euskadi, 1995.

Argueira Miranda, M.A.: Carácter revolucionario del cooperativismo. Buenos Aires: Intercoop Editora Cooperativa Limitada, 1965.

25 Miranda, José Eduardo, «El buen gobierno de las Sociedades Cooperativas y la gestión bajo los valores del Cooperativismo: una reseña didáctica», en Boletín de la Asociación Internacional de Derecho Cooperativo, n. ${ }^{\circ} 38$. Universidad de Deusto: Bilbao, 2004, p. 253.

26 Ibid., p. 254.

27 Aranzadi, 1995, 298. 
Borgadus, E.S.: Principios y problemas del cooperativismo. México D.F.: Libreros Mexicanos Unidos, 1964.

Hunter, James C.: O monge e o executivo. São Paulo: Sextante. 2004.

LASSERRE, G.: El hombre cooperativo. Buenos Aires: Intercoop Editora Cooperativa Limitada, 1980.

MACEDO, Ivanildo Izaias de e outros: Aspectos comportamentais da gestão de pessoas, 9 ed. Rio de Janeiro: Editora da FGV, 2007, p. 109.

MIRANDA, José Eduardo de Miranda: De la crisis de identidad al rescate de la génesis del cooperativismo. Madrid: Dynkinson, 2012.

MIRANDA, José Eduardo: «El buen gobierno de las Sociedades Cooperativas y la gestión bajo los valores del Cooperativismo: una reseña didáctica», en Boletín de la Asociación Internacional de Derecho Cooperativo, n. ${ }^{\circ} 38$. Universidad de Deusto: Bilbao, 2004.

Ostrower, F.: Criatividade e processos de criação. Petrópolis: Vozes, 1978.

Ramírez Baracaldo, B.: Teoría y doctrina de la cooperación. Bogotá: Ciudec, 1989.

Rosembus, T.: «El valor del socio en la cultura cooperativa», en Boletín de la Asociación Internacional de Derecho Cooperativo, 36, Bilbao. Universidad de Deusto, 2002. 